\title{
Evaluation of the state-of-the-art in informatics in glucometers
}

O. Ajai, A. Tiwari, J.R. Alcock

School of Applied Sciences, Cranfield University,

Cranfield, Bedfordshire, MK43 0AL, UK.

o.ajai@cranfield.ac.uk

Keywords: Glucometers, Informatics, Diabetes, Advice

\section{Abstract}

This review evaluated the level of informatics in glucometers through an assessment of the quantity and types of information and advice provided to users. Manufacturer websites were investigated and the characteristics of glucometers were examined. 100 glucometers from 27 manufacturers were analysed. Many glucometers contained simple informatics features and 5 also contained on-device graphing features for users to monitor trends. Some manufacturers have extended informatics via external software. A small number of glucometers provided knowledge for the user by, for example, simple embedded decision support protocols. However, it is suggested that glucometers could better serve as primary care devices through the incorporation of more decision support directly on the device. 


\section{Introduction}

The change in emphasis from acute to primary care is producing a requirement for a new generation of medical monitoring devices. As well as the technical challenges that will need to be overcome, informatics challenges must be met, if device users are to obtain full services from such devices. In particular, problems with future point-ofcare devices for medical use may result from the inaccuracy, poor transmission, garbled reception or misinterpretation of the information they are designed to provide [1]. It is useful therefore, to assess the state-of-the-art in informatics for current medical monitoring devices, of which the most sophisticated and widely used example is the blood glucometer. This paper represents such an assessment, along with a comparison of the state-of-the-art with medical informatics models.

\subsection{Background on Diabetes}

Diabetes Mellitus is a chronic condition characterised by elevated blood glucose due to absolute or relative deficiency in insulin. In 2000, it was estimated that there were 171 million people in the world with diabetes [2]. The classic symptoms of diabetes include "increased urinary frequency (polyuria), excessive thirst (polydipsia), excessive hunger (polyphagia) and unexplained weight loss" [3] and these aid in its diagnosis along with a blood test. The World Health Organization (WHO) describes two main types [3].

Type 1 Diabetes (Insulin-Dependent Diabetes Mellitus) - is commonly found in children and adolescents in whom the pancreas is unable to produce sufficient insulin to metabolize glucose in the blood thus resulting in hyperglycaemia. Type 2 Diabetes (Non-Insulin-Dependent Diabetes Mellitus) - usually occurs in adults and is related to 
obesity and unhealthy diets. There is a third type which only affects pregnant women - Gestational Diabetes, but this is generally a temporary condition normally resolved 6 weeks after delivery. However the risk of developing Type 2 Diabetes in the future is increased.

\subsection{Monitoring Diabetes}

Patients with diabetes need to monitor their blood glucose regularly as there are risks associated with high blood glucose in the body including: eye damage, leading to blindness; kidney damage, leading to renal failure; nerve damage, leading to impotence and foot ulcers which may result in amputation (diabetes is the leading cause of non traumatic amputation); ketoacidosis, a high concentration of ketones in the body occurring when the body is unable to metabolize glucose for the cells, thus finding an alternative by using fat and proteins instead; heart disease; stroke; coma in extreme cases [4].

Blood glucose monitoring for patients can be done through regular visits to their health professional, however patients can also monitor their own blood glucose regularly with a glucometer or glucose meter.

\subsection{Glucometers}

Glucometers are designed for monitoring rather than for diagnosing diabetes. Some glucometers require coding, which refers to calibrating the meter to the batch of test strips being used as there is variation between batches of strips. If the glucometer is not calibrated, it will give an incorrect result [5]. 
The blood sample for the test is generally taken from the fingertip as this provides the most accurate result but it also causes the patient the most pain because the fingertips contain many nerve endings. Therefore some glucometers allow the testing of blood samples from other sites and this is known as alternative site testing (AST). The sites include "upper-arms, thighs, base of the thumb, stomach areas and forearms" [6 - 8].

A lancet is needed to obtain the blood sample and it has different settings to determine the depth to which the skin will be pierced, which helps in determining the size of sample obtained. Modern glucometers are able to analyze relatively small amounts of blood (minimum of $0.3 \mu \mathrm{L})$.

The test strips for glucometers vary and the latest ones available are designed to allow easier blood application. Some have a confirmation window that will provide assurance to the patient that a sufficient amount of blood has been provided [9].

Glucometers provide the blood glucose results in specified units: either mg/dL (which is the standard for USA) or mmol/L (which is the standard in UK, Canada and some European countries). Some glucometers have the capacity to store the results and some also have averaging features to aid users in gaining an overview of their blood glucose levels over periods of time.

\subsection{Aim}

The aim of this review was to ascertain the state-of-the-art in informatics for glucometers. The level of informatics available on the device was analysed and the quantity and types of information provided to users were investigated. The review describes the methodology used, gives the results obtained from that methodology, discusses the findings and compares them against the state-of-the-art in informatics. 


\section{Methodology}

A key word search was carried out to identify different types of commercially available glucometers. An on-line resource, for example [10], and the British National Formulary (BNF) were of particular assistance.

Glucometer manufacturers' websites were investigated to obtain the details of the glucometers they supplied. In total, information on 100 glucometers, available between the years 1998 and 2008, was obtained. This represented 27 manufacturers. The list of glucometers assembled for this review was not exhaustive; however it included popular brands available, for example, Abbott, Bayer, LifeScan and Roche Diagnostics.

The operating manuals of $72 \%$ of glucometers were available online or after request from manufacturers. There were no manuals for $28 \%$ of devices by 14 manufacturers/distributors thus resulting in incomplete information. However, a short description was normally provided by the manufacturer for those glucometers that did not have online manuals. The manuals contained detailed instructions on how to operate the glucometer as well as its features and specification.

The manuals were analysed to obtain glucometer specifications and operating details. A categorization scheme was drawn up to investigate whether glucometers possessed particular informatics features. Where glucometers were supplemented with "offdevice" data management software, the capabilities of the software were analysed. The results obtained are presented below in five groups: information before testing, testing, on-device data manipulation after testing, off-device data-management software capabilities and "advice" given by the glucometer. 


\section{Results}

\subsection{Information before testing}

$67 \%$ of devices reviewed required coding and $27 \%$ did not. Information about coding could not be obtained for $6 \%$ of devices.

$19 \%$ of devices had the option of setting reminders for the patient to test, therefore showing many manufacturers have not considered this as an essential feature to incorporate.

$62 \%$ of devices displayed an error message indicating that the glucometer was not operating in the right temperature range. In addition, $37 \%$ of devices also displayed a thermometer symbol on their screen to indicate that it was not operating in the right temperature range.

\subsection{Testing}

The time taken to generate results on devices was compared and the range was between 3-50 seconds. Some devices provided a time range for the time taken to obtain the result and for the purpose of data analysis this was adjusted to the maximum time given. The shortest time taken to obtain test results was 3 seconds and that was seen in 1 device - "Evolution" (Infopia). The majority of the devices (3 0\%) generated their result in 5 seconds, followed by $20 \%$ of devices generating their result in 10 seconds and $15 \%$ of devices generating results in 7 seconds (see Figure 1). The maximum time recorded was 50 seconds on "Prestige IQ" (Home diagnostics). 


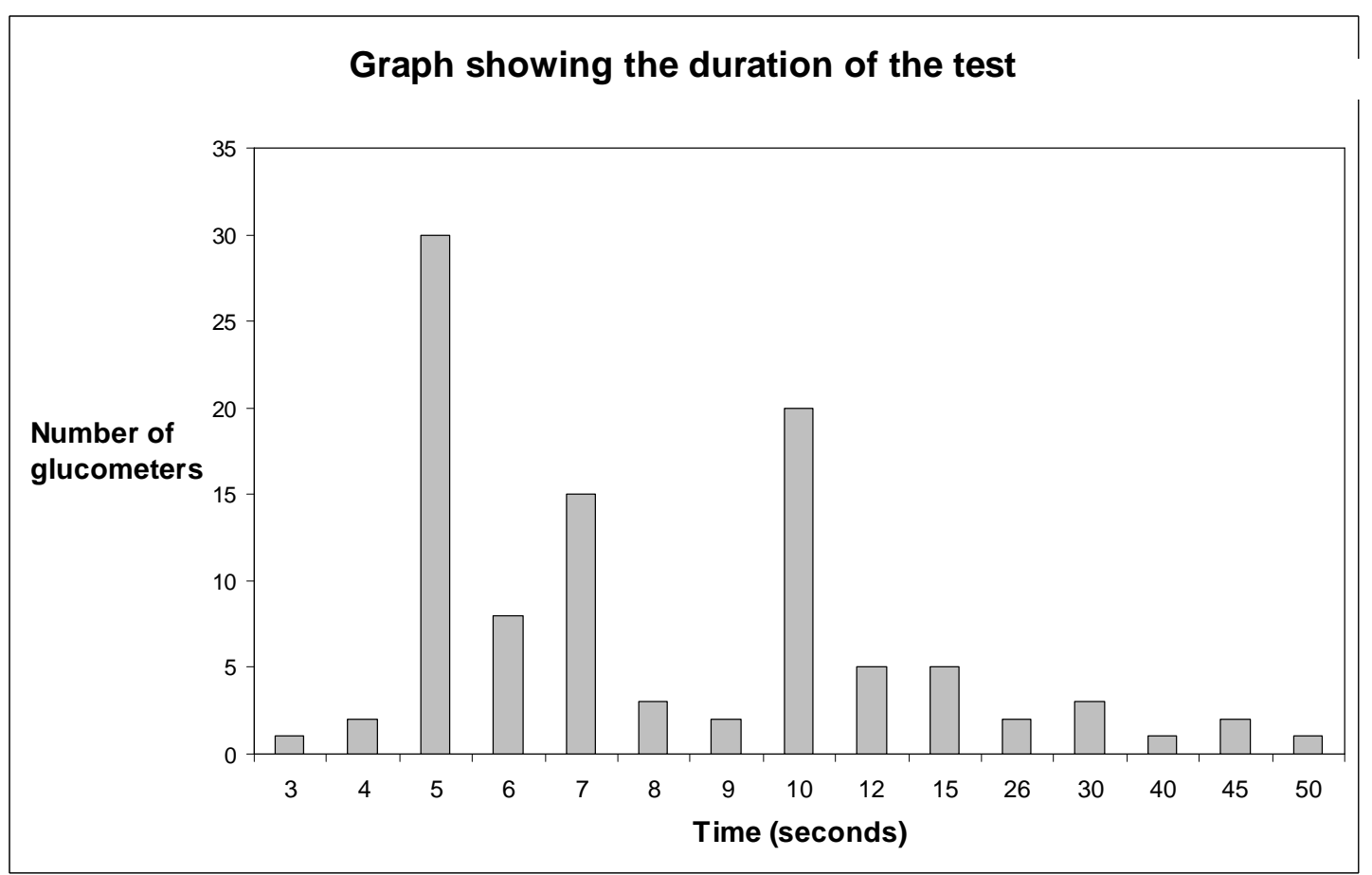

Figure 1 - Duration of the test.

\subsection{On-device data manipulation after testing}

$20 \%$ of devices allowed results to be flagged or marked with a symbol in order to associate a particular event to the result.

$5 \%$ allowed users to add a comment to annotate the result. The glucometers provided predefined comments for users to select from. Table I shows examples of the comments available. Normally, the comments were found in a single list. However, the "OneTouch UltraSmart" (Life Scan) glucometer divided them into four categories - Food, Health, Exercise and Medication. 
Table 1 - A summary of comments provided on five glucometers to annotate the results.

\begin{tabular}{|c|c|}
\hline Glucometer & Available Comments \\
\hline $\begin{array}{l}\text { Accu-Chek Complete } \\
\text { (Roche Diagnostics) }\end{array}$ & $\begin{array}{l}\text { Before Meal, After Meal, Fasting, Snack, Feel } \\
\text { Hypo., Before Exercise, After Exercise, Illness, } \\
\text { Invalid Test, Other's Result, User Defined, } \\
\text { Stress, L1 Control, L2 Control, Oral Medication }\end{array}$ \\
\hline $\begin{array}{l}\text { OneTouch Profile } \\
\text { (LifeScan) }\end{array}$ & $\begin{array}{l}\text { Fasting, pre breakfast, after breakfast, pre noon } \\
\text { meal, after noon meal, pre dinner, after dinner, } \\
\text { different food, bedtime, during night, pre } \\
\text { exercise, after exercise, illness, hypoglycemia, } \\
\text { other }\end{array}$ \\
\hline $\begin{array}{l}\text { OneTouch UltraLink } \\
\text { (LifeScan); } \\
\text { OneTouch Ultra2 } \\
\text { (LifeScan) }\end{array}$ & $\begin{array}{l}\text { No comment, Stress, Not enough food, Illness, } \\
\text { Too much food, Feel hypo, Mild exercise, } \\
\text { Menses (period), Hard exercise, Vacation, } \\
\text { Medication, Other }\end{array}$ \\
\hline $\begin{array}{l}\text { OneTouch UltraSmart } \\
\text { (LifeScan) }\end{array}$ & $\begin{array}{l}\text { Food Comments - BefBrkft, Aft Brkft, Bef } \\
\text { Lunch, Aft Lunch, Bef Dinner, Aft Dinner and } \\
\text { Night } \\
\text { Health Comments - Stress, Feel Hypo, Illness, } \\
\text { Menses, Vacation, Other } \\
\text { Exercise Comments - Before, During, After } \\
\text { Medication - None }\end{array}$ \\
\hline
\end{tabular}


$81 \%$ of devices calculated an average of the results recorded. Devices varied in the number of days over which an average could be calculated, and in how many variations of averages were permitted. The maximum number of variations of the average was 6 types i.e. 7, 14, 21, 28, 60 and 90-day averages. Figure 2 plots the amount of glucometers against the number of types of average which they were able to display.

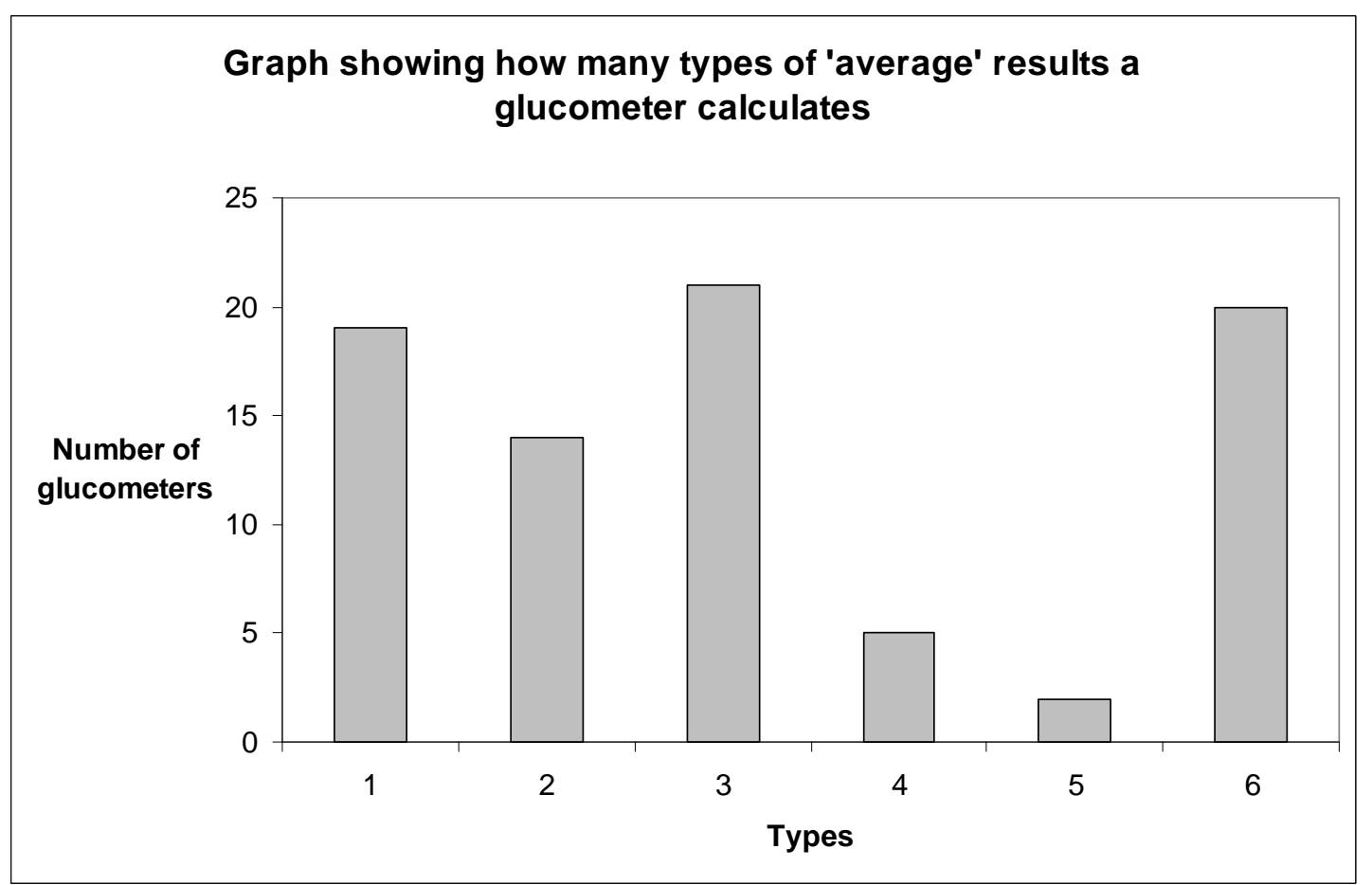

Figure 2 - Types of averages calculated on glucometers.

The majority of devices $(21 \%)$ calculated three types of averages, with $15 \%$ that calculated 7, 14 and 30-day averages, 2\% that calculated 7, 14 and 28-day averages and $3 \%$ that calculated 14, 30, 90-day averages. One device, "Eclipse" (Infopia) allowed the patient to set 3 unique averages. $20 \%$ calculated six types of averages and $19 \%$ calculate 1 type of average. Glucometers calculating six types of averages were limited to four manufacturers: CardioCom, Diabetic Supply of Suncoast Inc., Diagnostic Devices Inc. and TaiDoc. 
$79 \%$ of devices allowed results to be downloaded to a computer, thus showing that a majority of manufacturers have considered this as a feature to include. $11 \%$ did not allow results to be downloaded. Manufacturer's information for downloading could not be obtained for $10 \%$ of glucometers.

\subsection{Off-Device data-management software}

$51 \%$ of glucometers were found to be available with data management software, which extended data analysis "off device".

Of these software packages, $90 \%$ required data to be transferred via a USB cable; $10 \%$ used infra-red connection.

$80 \%$ highlighted outliers in the blood glucose concentration either within a "logbook" or in graphs generated.

$90 \%$ allowed the generation of reports, the numbers of types of reports ranging from 5 to $12.40 \%$ allowed reports to be emailed, however printing or faxing of results were offered as an alternative.

$60 \%$ allowed the importing of extra data in addition to the data downloaded from glucometers. $30 \%$ did not have import facilities. Manufacturer's information could not be obtained for the remaining $10 \% .70 \%$ allowed exporting of data to another application whereas $20 \%$ did not have export facilities. Manufacturer's information could not be obtained for the remaining $10 \%$.

All the software packages reviewed the calculated averages. $70 \%$ had further statistical analysis capabilities such as standard deviation, minimum and maximum. 


\subsection{On-device and off-device "advice"}

$77 \%$ of devices displayed a comment for example "hi" or "lo" whenever the blood glucose result was not within the specified measuring range of the device. All manuals included definitions of these comments. The measuring range of 53\% of the glucometers was between 20 and $600 \mathrm{mg} / \mathrm{dL}$ (or $1.1-33.3 \mathrm{mmol} / \mathrm{L}$ ). For $23 \%$, manufacturer's information could not be obtained. Within these 77 devices, $73 \%$ specified in the manual that users needed to repeat their test to confirm the results, whenever these warning comments were displayed.

Certain glucometers also provided warnings for actual, measured values of blood glucose that were particularly high or low. When a user's blood glucose was lower than a specific range, "OneTouch Profile" (Life Scan) and "OneTouch UltraSmart" (LifeScan) glucometers displayed a message: "Do you need a snack"?

"Danger" or "Call Dr" was displayed on "OneTouch Profile" (LifeScan) when a user's blood glucose was higher than the specified range. The other glucometers displayed a message related to checking ketones in the blood.

"Accu-Chek Complete" (Roche Diagnostics) displayed a message while the user was carrying out a test, known as the tip of the day, which sometimes provided a reminder or supplied information to the user about managing their diabetes.

$21 \%$ of glucometers displayed messages providing advice on further action to take based on their blood glucose result. For example, a message to check for ketones was displayed. $52 \%$ did not provide further comments. Manufacturer's information could not be obtained for the remaining $27 \%$.

"GlucoTel" (BodyTel Scientific Inc), (not yet commercially available) was specified to incorporate human interaction in terms of the provision of advice. Once results are transmitted and stored in the user's electronic patient health record held on an external 
database, health professionals could set the option of being alerted (via email, text message or fax) should the user's blood glucose result be extreme. They could then provide the most appropriate advice to the user.

$74 \%$ of glucometers were found to provide information explaining the messages that were displayed on the glucometers. These included error messages. This information was always placed in the manual. Manufacturer's information could not be obtained for the remaining $26 \%$. $25 \%$ of glucometers surveyed had manuals that provided multiple reasons for individual error messages, leaving some interpretation of the message to the user. For error messages, the glucometers provided advice to the user regarding the actions to take and in some cases users were advised to redo their test. Error messages shown on the glucometers included issues relating to the testing process. For example, faulty test strips, the size of the blood sample provided and the temperature at which the test was carried out. Other error messages were related to general problems with the glucometer which would make it unusable. Certain general messages were provided in a pictorial form e.g. a battery symbol represented low battery on the glucometer and a thermometer symbol indicated that the environmental temperature for the glucometer was not in the right range.

\section{Discussion}

\subsection{Information provided to users}

Coding or calibration of a meter prior to testing is an extra step in blood glucose testing and glucometers that are automatically coded reduce the error that may occur in blood glucose results. $67 \%$ of the glucometers reviewed required a coding step. 
Newer glucometer models have been developed with automatic coding features. However neither set-up provided the user with an estimate of the calibration error.

As testing of glucometers indicates that glucometer inaccuracy is so high that they cannot yet be used as a diagnostic test, this lack of error estimation is a serious limitation on their informatics capabilities [11].

"Prodigy Eject" (Diagnostic Devices Inc) and "Assure Pro" (ARKRAY) reminded users to carry out a control test which enabled patients to check that the glucometer and strips were functioning correctly. This was a necessary step that needed to be carried out occasionally to ensure that the results obtained from the glucometer were accurate and the patient was not misinformed, which may lead to incorrect medication being administered leading to side effects. Control tests were also suggested from the manual when unexpected results were obtained from the glucometer.

The proportion of glucometers reviewed providing an alarm function was 19\%. This is particularly important as patient non-compliance with monitoring regimes is an important barrier in the move from acute to primary care. In particular, users with busy or unpredictable lifestyles, reduced memory function or those yet to establish a monitoring routine would benefit from improved alarm functions.

Many of the reviewed glucometers incorporated a feature such that the measurement process started automatically once a sufficient blood sample had been provided, otherwise an error message was displayed by the glucometer.

A general trend was seen in the provision of advice to users, through the manual, of possible post-test actions to take. $73 \%$ of glucometers instructed users to redo their test when unexpected results were obtained. Retests were also suggested whenever there were no factors that could be affiliated to the result obtained by user action, (see below) (for example, the glucometer may have produced a low result, but the user had 
recently had a meal). $17 \%$ of glucometers displayed a message regarding checking for ketones. 1 glucometer "Precision Xtra" (Abbott) provided the facility to test for ketones, allowing the user ability to immediately act on the advice.

\subsection{User interaction with the results}

$20 \%$ of glucometers allowed results to be flagged on the device. The intention of the flags was to highlight particular results so that when data was reviewed, users would be able to discuss with their health professional, possible contributory factors, such as food intake or exercise. However, the flag systems generally consisted of one letter symbols and so could not provide detailed descriptions of factors. 6 glucometers had added to the utility of flags by allowing their association with meals i.e. a result could be flagged as occurring before or after a meal. For these glucometers, flagged results were also not included in the calculation of averages of results. This was a way of excluding outliers and the bias they may have introduced into users' averages. However, this assumes that the correct course of action would be to exclude these flagged events when calculating a users' average data.

Averaging of results was a simple and quick way of data manipulation, possessed by $81 \%$ of glucometers. This was the most advanced form of data manipulation seen on most glucometers, although "OneTouch UltraSmart" (LifeScan), "WaveSense Keynote" (Agamatrix), "WaveSense Keynote Pro" (Agamatrix), "Presto" (Agamatrix) and "Accu-Chek Complete" (Roche Diagnostics) provided on-device functionality to visualise mini graphs to observe trends in the results.

Predefined comments by manufacturers could be added to results to annotate them. Notably, "Accu-Chek Complete" (Roche Diagnostics) provided software that allowed comments to be modified. Incorporation of this approach into glucometers in general 
would allow both users and health professionals to monitor and assess how the user's lifestyle was influencing their blood glucose and the advice that could be given to the user on how to improve their health.

In order to improve functionality, $74 \%$ of manufacturers have developed off-device software to manipulate results obtained from the glucometer. The off-device software possessed certain common features across manufacturers, such as graphing functionality to visualise trends and an electronic "logbook".

All glucometers reviewed had a memory capacity. This functionality reduced the need for users to otherwise manually log their blood-glucose data.

\subsection{Glucometers and Informatics}

Several informatics models exist which could be applied to glucometers. For example, Georgiou (2002) [12], described a health informatics model comprising data, information and knowledge where data was at the lowest level of the hierarchy and has limited meaning on its own. Information provided context for managing data, while knowledge provided guidance for the cause of action to take based on insight and experience. In terms of conformity with such a health informatics model, all glucometers evaluated displayed data. To provide information about the result, $81 \%$ of glucometers incorporated an average function that allowed possible trends in the data to be identified, but this was not automatically displayed. Cases where information was provided automatically were when extreme results were displayed (e.g. Hi or Lo); and when tests were carried out in extreme conditions (i.e. a temperature symbol was displayed).

Some glucometers also possessed an aspect of knowledge although this was limited to comments when an extreme result was obtained. To further improve the quality of 
knowledge provided, glucometers would need to be embedded with decision support which would incorporate results with known facts about diabetes thus aiding users in managing their health. For example, glucometers could include a rule-based decision framework as described by Manjanatha et al. (2007) [13]. Manjanatha noted that current medical devices did not integrate user care information with device-generated data, therefore advice could be provided by the device. Though the authors came to these conclusions for devices offering continuous monitoring, which provided information based on changing conditions of users, the information in this paper indicates that this conclusion could be extended to stand-alone devices such as glucometers.

\section{Conclusion}

The paper has described the results from an evaluation of commercial glucometers. It has highlighted the main informatics features available. Many glucometers provide information through their manuals. However, limited data processing and real-time decision support, was found. It is suggested that glucometers could better serve as primary care devices by incorporating more decision support directly on the device.

\section{References}

[1] Meier, F.A. and Jones, B. A. Point-of-care testing error: Sources and amplifiers, taxonomy, prevention strategies, and detection monitors, Arch. Pathol. Lab. Med., Oct. 2005, vol. 129, no. 10, pp. 1262-1267. 
[2] Definition and diagnosis of Diabetes Mellitus and intermediate hyperglycemia Report of WHO/IDF Consultation [Internet]. World Health Organization 2006 [cited 2008 May 20]. Available from:

http://www.who.int/diabetes/publications/Definition\%20and\%20diagnosis\%20of\%20

diabetes new.pdf

[3] Types of Diabetes [Internet]. World Health Organization 2008 - [cited 2008 Jun 26]. Available from:

http://www.who.int/diabetesactiononline/diabetes/basics/en/index 1 .html

[4] Complications of Diabetes [Internet]. World Health Organization 2008 [cited 2008 Jul 7]. Available from:

http://www.who.int/diabetesactiononline/diabetes/basics/en/index3 .html

[5] Proud, L.J. and Bayer Healthcare LLC, 2004. Miscoding:Impact on the Accuracy of Blood Glucose Monitoring Systems. Available from http://www.bayerdiabetes.com/Print/Miscoding.pdf

[6] Cembrowski, G. Alternate Site Testing: First Do No Harm. Diabetes Technology \& Therapeutics, 2002, 4(1).

[7] Lucidarme, N., Alberti, C., Zaccaria, I., Claude, E. and Tubiana-Rufi, N. Alternate-Site Testing Is Reliable in Children and Adolescents With Type 1 Diabetes, Except at the Forearm for Hypoglycemia Detection. Diabetes Care, 2005, 28(3), pp. 710-7 11.

[8] Painless Glucose Monitors [Internet]. American Diabetes Services. 2004 [cited 2008 Jun 9]. Available from:

http://www.americandiabetes.com/AlternateSite.htm 
[9] Prodigy Autocode - product description [Internet]. Diagnostic Devices Inc. 2008 - [cited 2008 Jun 9]. Available from:

http://www.prodigymeter.com/prodDetails.cfm?itemid=213

[10] Blood glucose meters [Internet]. Mendosa, D., The diabetes monitor. 2008 [cited 2008 Jun 16]. Available from: http://www.diabetesmonitor.com/meters.htm

[11] Slingerland, R.J. and Miedema, K. Evaluation of portable blood glucose meters. Problems and recommendations, Clinical Chemistry and Laboratory Medicine, 2003, vol. 41, no. 9, pp. 1220-1223.

[12] Georgiou, A. Data, information and knowledge: the health informatics model and its role in evidence-based medicine, Journal of Evaluation in Clinical Practice 2002, 8, 2, pp.127-130.

[13] Manjanatha, S.; Bestavros, A.; Gaynor, M.; Moulton, S.A. Rule-based decision framework for medical sensor networks, Joint Workshop on High Confidence Medical Devices, Software, and Systems and Medical Device Plug-andPlay Interoperability (HCMDSS-MDPnP); 2007 June 25-27, Cambridge MA, IEEE, pp.192-193. 


\section{ACKNOWLEDGMENT}

The authors wish to acknowledge the EPSRC and the Cranfield IMRC for the provision

of funding. 